\title{
Bounds on Benefits and Harms of Adding Connections to Noncooperative Networks
}

\author{
Hisao Kameda \\ University of Tsukuba, Tsukuba Science City 305-8573, Japan \\ kameda@is.tsukuba.ac.jp \\ http://www.osdp.is.tsukuba.ac.jp/ ^hkameda/
}

\begin{abstract}
In computer networks (and, say, transportation networks), we can consider the situation where each user has its own routing decision so as to minimize noncooperatively the expected passage time of its packet/job given the routing decisions of other users. Intuitively, it is anticipated that adding connections to such a noncooperative network may bring benefits at least to some users. The Braess paradox is, however, the first example of paradoxical cases where it is not always the case. This paper studies the bounds on the degrees of coincident cost improvement (benefits) and degradation (harms) for all users by adding connections to noncooperative networks. For Wardrop networks (noncooperative networks with infinitesimal users), the degree of benefits for all users can increase without bound by adding connections whereas no Wardrop network has been found for which the degree of harms can increase without bound for all users. In contrast, for Nash networks (noncooperative networks with a finite number of users), the degrees of both benefits and harms can increase without bound for all users. On the other hand, we see that, for some category of Wardrop networks, adding connections to them can bring neither benefits nor harms to all users, and that, for some homogeneous networks, adding connections to them can never bring benefits to all users under any static policy including cooperative and noncooperative ones.
\end{abstract}

Keywords: Braess paradox, Wardrop equilibrium, Nash equilibrium, coincident cost degradation and improvement, computer and communication networks, distributed computer systems, network routing, shortest path routing, source routing, static load balancing.

\section{Introduction}

There exist networks and systems wherein a number of independent users share and compete for resources. For example, communication networks like the Internet consist of a finite number of links, and of flows of packets each of which is issued by an independent user and flows through the links. Distributed computer systems like GRIDs [1] consist of computers widely distributed and interconnected by communication networks, and of flows of jobs each of which is issued by an independent user. 
We can consider communication networks where each infinitesimal user has its own routing decision so as to minimize the expected passage time of its packet from the origin and the destination given the routing decisions by other users. The situation where every infinitesimal user has attained its optimization given the decision of other users and would not unilaterally deviate its routing decision is called a Wardrop equilibrium, a Nash equilibrium with infinitesimal players. We call here such a noncooperative network in a Wardrop equilibrium a Wardrop networks. In computer networking, some shortest path routing protocols may bring about situations close to Wardrop equilibria. It would be anticipated that users' benefits would increase by adding connections to a network, and thus increasing the degree of freedom in choices to each user. This is not always the case, however, as first exemplified in the Braess paradox [2].

The famous Braess paradox shows that adding connections (links) to a network may sometimes degrade the cost for all users in a Wardrop equilibrium. The Braess paradox attracted the attention of many researchers and a lot of work has been accumulated, e.g., $[3,4,5,6,7,8,9,10,11,12,13,14,15,16]$. Coincident cost degradation for all users by adding connections to a network looks paradoxical, and is called the paradox. It seems that, since its discovery, the paradox has been studied mostly with respect to Wardrop equilibria. It also seems that the topologies of concrete networks examined with respect to the paradox have been similar to or centered on that of the Braess network.

On the other hand, we can consider networks where users are classified into a small number of groups, each of which optimizes its cost non-cooperatively. The situation where, in such a scheme, every user has attained its optimization given the decision of other users and would not unilaterally deviate its decision is called a Nash equilibrium. We call here such a noncooperative network in a Nash equilibrium a Nash network. In computer networking, some source routing protocols may bring about situations close to Nash equilibria. Examples of paradoxes similar to Braess's in a Nash equilibrium have been found not only for networks of topologies similar to the Braess one $[17,18]$, but also for a network of another topology [19].

It seems that, except $[20,21]$, few studies have emphasized the estimation of how harmful the paradox can be, i.e., the worst-case degree of coincident cost degradation for all users by adding connections to a noncooperative network. Moreover, we have not seen the estimation of how beneficial the addition of connections to a noncooperative network can be, i.e., the best-case degree of coincident cost improvement by adding connections to a noncooperative network.

We consider networks with fixed numbers of vertices. It has been shown that the degree of coincident cost degradation by adding connections to Wardrop networks with one origin-destination pair is bounded [20,21]. On the other hand, it has been shown that the degree of coincident cost degradation by adding connections to Nash networks can increase without bound [22]. The present paper addresses the estimation of the best-case degrees of coincident cost improvement for all users by adding connections to Wardrop and Nash networks. We show that the degree of coincident cost improvement (benefits) by adding connections 
to both Wardrop and Nash networks can increase without bound. In contrast, for homogeneous distributed systems, no coincident cost improvement occurs by adding connections under any static load balancing policy including cooperative and noncooperative ones. Furthermore, in Wardrop equilibia for any distributed system, neither coincident cost degradation nor coincident cost improvement occurs by adding connections to the system.

Note, in passing, that each user of a network may have decisions on flow control, in addition to routing. In wireless networks, users may have power control. The concept of a Nash equilibrium is also discussed in flow control $[23,24,25,26,27,28]$ and in power control $[29,30,31,32,33]$, both of which are not addressed in this paper.

The outline of this paper is as follows. Section 2 presents the concepts around Pareto superiority, Braess-like paradoxes, and their degrees of magnitude. Section 3 gives the description of the Wardrop networks investigated, and presents the results. On the other hand, Section 4 examines networks with a topology different from that of the Wardrop networks, in particular, with that of distributed systems. Section 5 concludes this paper.

\section{A Measure Showing the Degree of the Paradox}

This paper uses a single scalar measure that shows the degree of Pareto superiority of a system state before adding connections to that after doing so, or the degree of paradoxes in Nash equilibria.

The Pareto superiority is defined as follows. Consider a system consisting of $n$ users (or players, decision makers), $1,2, \ldots, n$. User $i$ has its cost $C_{i}(S)$, in the system state $S$. Denote by $S^{a}$ and $S^{b}$ two different states of the system. $k_{i} \triangleq C_{i}\left(S^{a}\right) / C_{i}\left(S^{b}\right) . S^{b}$ is Pareto superior to $S^{a}$ iff $k_{i}>1$ for some $i$ and $k_{j} \geq 1$ for all other $j$. In particular, we say that $S^{b}$ is strongly Pareto superior to $S^{a}$ iff $k_{i}>1$ for all $i$. A state to which some other state is Pareto superior is Pareto inefficient. Thus, the Pareto superiority depends on the vector $\left(k_{1}, k_{2}, \ldots, k_{n}\right)$. It may, however, be convenient to express the degree of Pareto superiority, using a single scalar measure. It is required that the measure should clearly reflect Pareto superiority. If $k_{\min }>1$, the state $S^{b}$ is (strongly) Pareto superior to $S^{a}$, and if $k_{\min }<1$, the state $S^{b}$ is Pareto indifferent or superior to $S^{a}$. Thus, the measure $k_{\min }$ may be used as a primary measure of Pareto superiority. In contrast, for example, a measure based on a certain average or on a product of all $k_{i}$ cannot satisfy the above requirement, but may be used as a secondary measure for tie-breaking the case where $k_{\min }=1$.

It would be anticipated that in a system state where each user has more freedom of choice than in another state, at least one user should enjoy higher utility than in the latter state. But, as the famous Braess paradox shows, it is not always the case for noncooperative systems. Thus, a Nash equilibrium of a system with less freedom may be Pareto superior to that with more freedom, which is called paradox. We, therefore, use the measure of Pareto superiority as the measure of the degree of the paradox, i.e., the coincident cost degradation 
for all users by adding connections. The measure of the degree of coincident cost improvement for all users after due by adding connections can be considered in a similar way.

\section{$3 \quad$ Wardrop Networks}

\subsection{Assumptions on Wardrop Networks}

Wardrop networks considered here consist of one origin and one destination and some relay nodes, some pairs of which a one-way link connects. One of the simplest networks is the general Braess network (Fig. 1) discussed later. There are a number of paths each of which connects the origin and the destination through a different series of links. The cost of a path is the sum of the cost of each link in the path. Infinitely many infinitesimal users send their packets through the network. Each user chooses a path of the minimum cost. The choice of a single infinitesimal user has only a negligible impact on the cost of each link. The situation where no user can reduce his/her cost by unilaterally choosing another path is a Wardrop equilibrium, an infinitesimal-user version of a Nash equilibrium.

It is assumed that the cost of each link is a non-decreasing function of the total flow, i.e., the rate of packets through the link. In a Wardrop equilibrium of the networks with only one pair of origin and destination, the costs of all users are identical. $C_{\mathrm{o}}$ and $C_{\mathrm{c}}$, respectively, denote the costs of users of a Wardrop network before and after adding connections to the network. Define $k=C_{\mathrm{c}} / C_{\mathrm{o}}$. Then, $k$ expresses the degree of cost change for all users by adding the connections. $k_{\min }$ of Section 2 reduces to $k$ here.
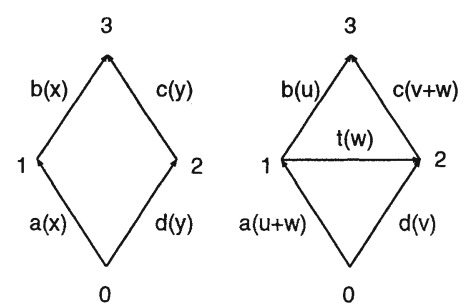

Fig. 1. General Braess network. Left: The network before link 1-2 is added. Right: The network after link 1-2 is added.

The Braess network consists of four nodes: one origin, one destination, and two relay nodes (Fig. 1). Each user flows through one of the paths. Before adding connections, the network has two paths, $0-1-3$ and $0-2-3$, each of which contains two links, the origin to one relay (0-1 or $0-2)$ and the relay to the destination (1-3 or 2-3), respectively. After adding connections, i.e., a one-way link connecting 
two relays (1-2), the network has three paths including the new path (0-1-2-3) connecting the origin, one relay, the other relay, and the destination. Each user flows through one of the paths. In the original Braess network, the cost of each link is a linear function of the amount of the flow through the link [2].

This paper also considers the networks, called general Braess networks, that have nonlinear link cost functions. If we denote by $\eta$ the flow of each link, the costs of links $0-1,1-3,2-3,0-2$, and 1-2 are, respectively, denoted by $a(\eta), b(\eta)$, $c(\eta), d(\eta)$, and $e(\eta)$. The total flow through the network is denoted by $X$. Denote the amounts of the flows through paths $0-1-3$ and $0-2-3$, respectively, by $x$ and $y$ before adding link 1-2. Denote the amounts of the flows through paths $0-1-3$, $0-2-3$, and $0-1-2-3$, respectively, by $u, v$, and $w$ after adding link $1-2$. Denote by $X$ the total flow, and, thus, $x+y=X=u+v+w$. The cost of links $0-1,1-3,2-3$, and $0-2$, are, respectively, $a(x), b(x), c(y)$, and $d(y)$ before adding link 1-2. The cost of links $0-1,1-3,2-3,0-2$, and 1-2 are, respectively, $a(u+v), b(u), c(v+w)$, $d(v)$, and $t(w)$ after adding the link.

For the original Braess network, $a(\eta)=c(\eta)=10 \eta, b(\eta)=d(\eta)=\eta+50$, $t(\eta)=\eta+10$, and $X=6$, which result in $C_{\mathrm{o}}=83$ and $C_{\mathrm{c}}=92$, and thus $k=C_{\mathrm{c}} / C_{\mathrm{o}}=1.1084 \ldots$ [2]. Recall that $k$ shows the degree of cost change by adding link 1-2. $k>1$ means coincident cost degradation. In the above case, it is about $11 \%$ degradation.

By general Cohen-Kelly networks, we mean a subset of general Braess networks for which the costs of links $0-1$ and 2-3, are, respectively, $a(\eta)=\alpha /(a-\eta)$ and $c(\eta)=\alpha /(a-\eta)$ for $0 \leq \eta<a$ and for which the costs of links 1-3 and $0-2$, are, respectively, $b(\eta)=d(\eta)=b$ for any $\eta>0$ with $t(\eta)=t$ (constant). Cohen and Kelly [12] considered a network of this type for which $\alpha=1, a=\phi$, $b=2, t=1$, and $X=2 \lambda$. They showed that $C_{\mathrm{o}}=1 /(\phi-\lambda)+2<3=C_{\mathrm{c}}$, i.e., $1<k<3 / 2$, assuming that $2 \lambda>\phi-1>\lambda>0$, which is a paradox. In the above case, it is less than $50 \%$ degradation.

As a general result on the Braess networks, it has been shown that the degree, $k$, of coincident cost degradation is bounded to 2 for the general Braess networks for which $a(\cdot)$ and $c(\cdot)$ are increasing and $b(\cdot), d(\cdot)$, and $t(\cdot)$ are non-decreasing $[20]$. Furthermore, as a general result on the Wardrop networks, it has been shown that $k$ is bounded to $\lfloor n / 2\rfloor$ for Wardrop networks that consist of $n$ vertices and have link costs each of which is a nondecreasing function of the flow through the link [21].

We define more special Braess networks that will be used in the next section.

- $[$ Model Cohen-Kelly network] Networks that are the same as general CohenKelly networks with and $b \geq \alpha /(a-X)+t$. Thus, $u=v=0$ and $w=X$. Then, the following relations hold.

$$
\begin{aligned}
C_{\mathrm{o}} & =\frac{\alpha}{a-X / 2}+b, \\
C_{\mathrm{c}} & =\frac{2 \alpha}{a-X}+t .
\end{aligned}
$$


We consider the case of $t=0$, in particular. Then, model Cohen-Kelly networks are described by the values of parameters $\alpha, a, b$, and $X$ that satisfy $0<X<a$ and $0<\alpha /(a-X) \leq b$.

- [Reduced Cohen-Kelly network] A subset of model Cohen-Kelly networks with $b=\alpha /(a-X)+t$. Thus, the following relation holds for $C_{\mathrm{c}}$ while $C_{\mathrm{o}}$ is given by (1).

$$
C_{\mathrm{c}}=\frac{2 \alpha}{a-X}+t=2 b-t=\frac{\alpha}{a-X}+b .
$$

We also consider the case of $t=0$, in particular.

\subsection{The Results}

Proposition 1. For every value of $k$, s.t. $0<k<2$, there exist model CohenKelly networks for which the measure $k$ is that value.

[Proof] The outline of the proof is given as follows. For the details, see [34].

Step 1) shows that $k$ depends only on $\rho(=X / a)$ and $Z(=b(a-X) / \alpha-1)$, i.e., $k=k(\rho, Z)$.

Step 2) sees that $k=k(\rho, 0)$ monotonically increases in $\rho$ with the range $1<k<2$ and the domain $0<\rho<1$. Thus, given $k$, s.t. $1<k<2$, the corresponding value of $\rho$ can be obtained and thus, with $Z=0$, the corresponding combinations of values of $\alpha, a, X$, and $b$, can be obtained, which describe model Cohen-Kelly networks that are also reduced Cohen-Kelly networks.

Step 3) sees that, given $\rho$, s.t. $0<\rho<1, k=k(\rho, Z)$ monotonically decreases in $Z$ with the range $0<k \leq 1$ and the domain $Z_{\rho} \leq Z$ where $Z_{\rho}=\rho /(2-\rho)>0$. Thus, given $k$, s.t. $0<k \leq 1$, the corresponding value of $Z$ can be obtained and thus, with given $\rho$, the corresponding combinations of values of $\alpha, a, X$, and $b$, can be obtained, which describe model Cohen-Kelly networks.

Steps 4) shows, by combining 2) and 3), that, for every value of $k$, s.t. $0<k<$ 2 , the corresponding combinations of values of $\alpha, a, X$, and $b$, can be obtained, which describe model Cohen-Kelly networks. Therefore, for every value of $k$, s.t. $0<k<2$, there exist model Cohen-Kelly networks that have the value of $k$.

From Proposition 1, we see that there exist Wardrop networks for which the degree of coincident cost improvement (benefits) for all users by adding connections to the networks can increase without bound. Therefore, we have the following property.

Theorem 1. The degree of coincident cost improvement for all users by adding connections to Wardrop networks can increase without bound.

\section{Noncooperative Load Balancing in Distributed Systems}

The previous section has shown that by adding connections to Wardrop networks with fixed numbers of vertices, the degree of coincident cost improvement 
can increase without bound, although the degree of coincident cost degradation cannot be over some bound.

This section gives a category of noncooperative networks [19], for which it has been shown that the degree of the Braess-like coincident cost degradation can increase without bound in Nash equilibria [22]. This category of networks presents models of distributed systems, like GRIDs [1]. Load balancing of jobs among nodes in distributed systems are regarded as routing in the equivalent networks $[35,36,37]$.

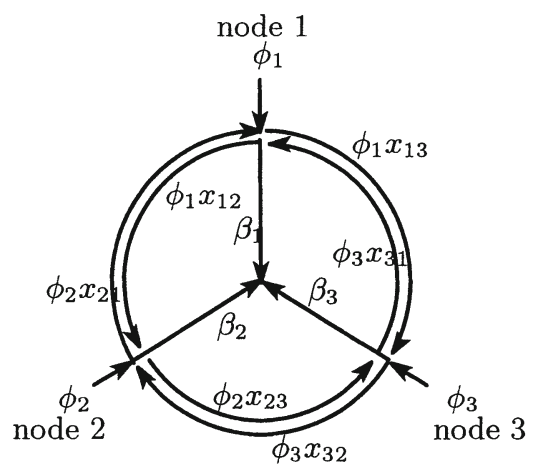

Fig. 2. The model of a distributed system for $m=3$. The symbols near each arrow express the rate of jobs that flow through the arrow. $\beta_{i}=\phi_{1} x_{1 i}+\phi_{2} x_{2 i}+\phi_{3} x_{3 i}, i=$ $1,2,3$.

\subsection{Assumptions on the Networks Equivalent to Distributed Systems}

A network equivalent to a distributed system consists of $m$ origins and one destination, with each origin being connected to the destination through one separate link, which is often called 'node' here. Denote the total flow of O-D pair $i$ is $\phi_{i}$.

Before adding connections, there is only one path for each O-D payer. After adding connections (see Fig. 2), some flow passes through the connections to forward to other nodes, out of the total flow $\phi_{i}$ that arrives at the origin $i$. Let the flow from origin $i$ to node $j$ be $\phi_{i} x_{i j}, i \neq j$. The amount of flow, $\phi_{i} x_{i i}$, goes to node $i$. Then, $0 \leq x_{i j} \leq 1, i, j=1,2, \ldots, m, \sum_{p} x_{i p}=1$. Denote the vector $\left(x_{i 1}, x_{i 2}, \ldots, x_{i m}\right)$ by $\boldsymbol{x}_{i}$ and the vector $\left(\boldsymbol{x}_{1}, \boldsymbol{x}_{2}, \ldots, \boldsymbol{x}_{m}\right)$ by $\boldsymbol{x} . \boldsymbol{x}$ denotes the strategy profile. The load balancing policies considered determine (only) $\boldsymbol{x}$, and, thus, are static in nature. Denote by $\boldsymbol{C}$ the set of $\boldsymbol{x}$ 's that satisfy the above constraints. The resulting flow $\beta_{i}$ through node $i, i=1,2, \ldots, m$, is

$$
\beta_{i}=\sum_{p} \phi_{p} x_{p i}
$$


Thus, the cost for the entire flow associated with the O-D pair $i$ is

$$
T_{i}(\boldsymbol{x})=\sum_{p} x_{i p} T_{i p}(\boldsymbol{x}),
$$

where $T_{i i}(\boldsymbol{x})=D_{i}\left(\beta_{i}\right)$, and $T_{i j}(\boldsymbol{x})=D_{j}\left(\beta_{j}\right)+G_{i j}(\boldsymbol{x})$, for $j \neq i$.

$D_{i}\left(\beta_{i}\right)$ denotes the cost of processing a packet/job at node $i$ in the case where the load on node $i$ is $\beta_{i} . G_{i j}(\boldsymbol{x})$ denotes the cost of sending a packet/job from origin $i$ to node $j$ in the case where the strategy profile is $\boldsymbol{x}$. It is assumed that, for all $i, j, k(i \neq j \neq k \neq i)$,

$$
G_{i j}(\boldsymbol{x})<G_{i k}(\boldsymbol{x})+G_{k j}(\boldsymbol{x}) .
$$

Thus, a job forwarded from a node is not to be forwarded again to another node.

\subsection{Wardrop Networks of This Category}

We consider an individual optimization scheme for the networks of this category. Each infinitesimal user seeks its own optimization and chooses the path of the minimum cost to send its jobs through. The situation where every infinitesimal user attains unilaterally its optimization given the decisions of other users is a Wardrop equilibrium. We call the network at a Wardrop equilibrium a Wardrop network as before. A Wardrop network in question is characterized as follows: Infinitesimal users choose the path of the smallest cost for each O-D pair. Thus, the Wardrop equilibrium $\overline{\boldsymbol{x}}$ after adding connections satisfies the following for all $i, j$ :

$$
\begin{aligned}
& T_{i j}(\overline{\boldsymbol{x}})=\min _{k}\left\{T_{i k}(\overline{\boldsymbol{x}})\right\} \text { for } \bar{x}_{i j}>0, \\
& T_{i j}(\overline{\boldsymbol{x}}) \geq \min _{k}\left\{T_{i k}(\overline{\boldsymbol{x}})\right\} \text { for } \bar{x}_{i j}=0 .
\end{aligned}
$$

That is, all the paths used have the equal cost for each O-D pair. We have the following property.

Theorem 2. The costs of all users neither degrade nor improve coincidently by adding connections to any Wardrop network of this category.

[Proof] Similarly as [35,38,37], after adding connections to a Wardrop network in question, nodes are one of the following:

(1) idle source $\left(R_{\mathrm{d}}\right)$ : The node sends jobs and does not process any jobs. That is, $\beta_{i}=0$.

(2) active source $\left(R_{\mathrm{d}}\right)$ : The node sends jobs and does not receive any jobs. But, the node processes a part of the jobs that arrive at the node. That is, $\phi_{i}>\beta_{i}>0$.

(3) neutral $(N)$ : The node processes jobs locally without sending or receiving jobs. That is, $\beta_{i}=\phi_{i}$. 
(4) $\operatorname{sink}(S)$ : The node receives jobs from other nodes but does not send out any jobs. That is, $\beta_{i}>\phi_{i}$.

That is, there does not exist such a node that both sends and receives jobs. Indeed, suppose that node $i$ sends jobs to node $j$ and receives jobs from node $k$. Then,

$$
\begin{gathered}
D_{i}\left(\beta_{i}\right) \geq D_{j}\left(\beta_{j}\right)+G_{i j}(\boldsymbol{x}), \\
D_{k}\left(\beta_{k}\right) \geq D_{i}\left(\beta_{i}\right)+G_{k i}(\boldsymbol{x})=T_{k i} .
\end{gathered}
$$

It is because, if (10) does not hold, processing jobs at node $i$ incurs the smaller cost than sending jobs to node $j$ from node $i$. Similarly for (11).

Then, from (10) and (7), we have $T_{k i}=D_{i}\left(\beta_{i}\right)+G_{k i}(\boldsymbol{x}) \geq D_{j}\left(\beta_{j}\right)+G_{i j}(\boldsymbol{x})+$ $G_{k i}(\boldsymbol{x})>D_{j}\left(\beta_{j}\right)+G_{k j}(\boldsymbol{x})=T_{k j}$. That is, $T_{k i}>T_{k j}$. Then, from (8) and (9), $x_{k i}=0$. That is, node $i$ does not receive jobs from node $k$, which contradicts the supposition.

Denote by $\bar{T}_{i}$ and $\hat{T}_{i}$, respectively, the cost of O-D pair $i$ before and after adding connections to the network in question. Recall that $D_{i}$ is nondecreasing for all $i$.

1) Assume that adding connections to a network in question brings about coincident cost degradation to all O-D pairs.

Suppose that there exists an idle or active node $i$ after adding connections, then $\bar{T}_{i}=D_{i}\left(\phi_{i}\right) \geq D_{i}\left(\beta_{i}\right) \geq \hat{T}_{i}$. That is, the O-D pair $i$ suffers no cost degradation. Thus, we see that there exists neither idle nor active node.

Then, since there exists neither idle node nor active node, there must exist no sink node. Only remain neutral nodes. That is, no coincident cost degradation occurs for all O-D pairs by adding connections to the network in question.

2) Assume that adding connections to a network in question brings about coincident cost improvement to all O-D pairs.

Suppose that there exists a sink node $i$, then $\bar{T}_{i}=D_{i}\left(\phi_{i}\right) \leq D_{i}\left(\beta_{i}\right)=\hat{T}_{i}$, which is no cost improvement for the O-D pair $i$. Thus, we see that there exists no sink node.

Then, since there exists no sink node, then there must exist neither idle node nor active node. Only remain neutral nodes. That is, no coincident cost improvement occurs for all O-D pairs by adding connections to the network in question.

\subsection{The Best-Case Degree of Coincident Cost Improvement by Adding Connections to Nash Networks}

A Nash network in question is described as follows: For each O-D pair, there is one decision maker, or a player, that strives to minimize the cost for the O-D pair, i.e., decision maker $i$ for O-D pair $i, i=1,2, \ldots, m$. Before adding connections, each decision maker has no choice since there is only one path for each O-D payer. After adding connections, decision maker $i(i=1,2, \ldots, m)$ chooses the amount of the flow to pass through the connections to forward to 
other nodes, out of the total flow $\phi_{i}$ that arrives at the origin $i$. Thus, within these constraints, decision maker $i(i=1,2, \ldots, m)$ chooses the values of $x_{i j}$, $j=1,2, \ldots, m$, to achieve optimization. Assume that each decision maker strives to optimize non-cooperatively the cost associated only with the corresponding O-D pair.

A Nash equilibrium is given by such $\tilde{\boldsymbol{x}}$ as satisfies the following for all $i$,

$$
T_{i}(\tilde{\boldsymbol{x}})=\min _{x_{i}} T_{i}\left(x_{i} ; \tilde{\boldsymbol{x}}_{-(i)}\right), \quad \text { such that }\left(x_{i} ; \tilde{\boldsymbol{x}}_{-(i)}\right) \in \boldsymbol{C},
$$

where $\left(x_{i} ; \tilde{\boldsymbol{x}}_{-(i)}\right)$ denotes an $m$-dimensional vector in which the element corresponding to $\tilde{x}_{i}$ has been replaced by $x_{i}$. $\tilde{\boldsymbol{x}}$ is called a solution for the above non-cooperative optimization.

Theorem 3. The degree of coincident cost improvement for all users by adding connections to Nash networks can increase without bound.

[Proof] 1) We first show this property even if $D_{i}(\cdot)$ and $G_{i j}(\cdot)$ are nondecreasing, $i, j(\neq i)=1,2, \ldots, m$.

Consider the following network with $m=2, \phi_{1}=\phi, \phi_{2}=\phi+\epsilon(\phi>0$, $\epsilon>0), \delta / D=D / \Delta \triangleq \kappa(0<\delta<D<\Delta)$,

$$
\begin{aligned}
D_{1}\left(\beta_{1}\right) & =D \text { (constant) } \\
D_{2}\left(\beta_{2}\right) & =\left\{\begin{array}{l}
\delta, \text { for } 0 \leq \beta_{2} \leq \phi, \\
\Delta, \text { for } \beta_{2}>\phi,
\end{array}\right. \\
G_{12} & =G_{21}=0 .
\end{aligned}
$$

$D_{1}, D_{2}, G_{12}$, and $G_{21}$ are nondecreasing. Then, clearly, $\tilde{\boldsymbol{x}}=\left(\tilde{x}_{11}, \tilde{x}_{12}, \tilde{x}_{21}, \tilde{x}_{22}\right)$ $=(0,1,1,0)$ is a Nash equilibrium after adding connections, and

$$
T_{1}(\tilde{\boldsymbol{x}})=\delta, \quad T_{2}(\tilde{\boldsymbol{x}})=D .
$$

Note, however, that, before adding connections,

$$
T_{1}(\tilde{\boldsymbol{x}})=D, \quad T_{2}(\tilde{\boldsymbol{x}})=\Delta .
$$

Therefore, $k_{1}=k_{2}=\kappa<1$, and both users 1 and 2 have coincident cost improvements by adding connections, and the best-case degree can increase without bound as $\kappa \rightarrow 0$.

2) We can easily show this property if we relax the condition that $D_{i}(\cdot)$ and $G_{i j}(\cdot)$ are nondecreasing, $i, j(\neq i)=1,2, \ldots, m$.

Consider the following network that is different from the above network only in the following. $0<\delta<D=\Delta$,

$$
\begin{aligned}
& D_{1}\left(\beta_{1}\right)=\left\{\begin{array}{l}
D, \text { for } 0 \leq \beta_{1} \leq \phi, \\
\delta, \text { for } \beta_{1}>\phi,
\end{array}\right. \\
& D_{2}\left(\beta_{1}\right)=D \text { (constant) }
\end{aligned}
$$


$D_{1}, D_{2}, G_{12}$, and $G_{21}$ are nonincreasing. Then, clearly, $\tilde{\boldsymbol{x}}=\left(\tilde{x}_{11}, \tilde{x}_{12}, \tilde{x}_{21}, \tilde{x}_{22}\right)$ $=(1,0,1,0)$ is a Nash equilibrium after adding connections, and

$$
T_{1}(\tilde{\boldsymbol{x}})=\delta, \quad T_{2}(\tilde{\boldsymbol{x}})=\delta .
$$

Note, however, that, before adding connections,

$$
T_{1}(\tilde{\boldsymbol{x}})=D, \quad T_{2}(\tilde{\boldsymbol{x}})=D,
$$

Therefore, $k_{1}=k_{2}=\delta / D<1$, and both users 1 and 2 have coincident cost improvements by adding connections, and the best-case degree can increase without bound as $\delta / D \rightarrow 0$.

3) Thus, we have seen that there exist Nash networks for which the degree of coincident cost improvement (benefits) for all users by adding connections to the networks can increase without bound. Therefore, we can say that the degree of coincident cost improvement for all users by adding connections to Nash networks can increase without bound.

\subsection{Homogeneous Distributed Systems}

As previously noted, it has been shown that, for homogeneous distributed systems, the degree of coincident cost degradation by adding connections can increase without bound in Nash equilibria [22]. That is, for any value of the ratio $k$, s.t. $k>1$, of coincident cost degradation by adding connections, there exists a homogeneous distributed system that has that value of the ratio in a Nash equilibrium. However, as to coincident cost improvement by adding connections to homogeneous distributed systems, we have the following property for all the static load balancing policies that determine $\boldsymbol{x}$.

Theorem 4. No static load balancing policy in homogeneous distributed systems after adding connections can bring about coincident cost improvement for all users over the situations before adding connections, if node cost functions are nondecreasing and convex.

[Proof] See Kameda [34].

Remark 1. From the above, we see that under any static load balancing policy (any policy that determines $\boldsymbol{x}$, not only noncooperatively but also cooperatively), it is impossible that all users have benefits coincidently from adding connections to homogeneous distributed systems. This result would give some insight into scheduling in GRID computing.

\section{Concluding Remarks}

The present paper has examined Wardrop and Nash networks that have fixed numbers of nodes and links. The results imply the following: For Wardrop networks, the degree of coincident cost improvement (benefits) can increase without 
bound by adding connections whereas there has been found no Wardrop network for which the degree of coincident cost degradation (harms) can increase without bound. On the other hand, for Nash networks, the degrees of both benefits and harms can increase without bound. In contrast, we have seen that some Wardrop network can have neither cost improvement nor cost degradation coincidently for all users by adding connection to it, and that some homogeneous network cannot have cost improvement coincidently for all users by adding connection to it under any static policy including cooperative and noncooperative ones.

\section{References}

1. Foster, I., Kesselman, C., eds.: The Grid: Blueprint for a New Computing Infrastructure. Morgan Kaufmann (1998)

2. Braess, D.: Über ein Paradoxen aus der Verkehrsplanung. Unternehmensforschung 12 (1968) 258-268

3. Samuelson, P.A.: Tragedy of the open road: Avoiding paradox by use of regulated public utilities that charged corrected Knightian tolls. J. Int. and Comparative Econ. 1 (1992) 3-12

4. Cohen, J.E., Horowitz, P.: Paradoxial behaviour of mechanical and electrical networks. Nature 352 (1991) 699-701

5. Murchland, J.D.: Braess's paradox of traffic flow. Transpn. Res. 4 (1970) 391-394

6. Frank, M.: The Braess paradox. Mathematical Programming 20 (1981) 283-302

7. Taguchi, A.: Braess's paradox in a two terminal transportation network. J. Oper. Res. Soc. of Japan 25 (1982) 376-388

8. Frank, M.: Cost effective links of ladder networks. Methods of Operations Research 45 (1984) $75-86$

9. Steinberg, R., Zangwill, W.I.: The prevalence of Braess's paradox. Transportation Science 17 (1983) 301-318

10. Dafermos, S., Nagurney, A.: Sensitivity analysis for the asymmetric network equilibrium problem. Mathematical Programming 28 (1984) 174-184

11. Dafermos, S., Nagurney, A.: On some traffic equilibrium theory paradoxes. Transpn. Res. B 18 (1984) 101-110

12. Cohen, J.E., Kelly, F.P.: A paradox of congestion in a queuing network. J. Appl. Prob. 27 (1990) 730-734

13. Calvert, B., Solomon, W., Ziedins, I.: Braess's paradox in a queueing network with state-dependent routing. J. Appl. Prob. 34 (1997) 134-154

14. Cohen, J.E., Jeffries, C.: Congestion resulting from increased capacity in singleserver queueing networks. IEEE/ACM Trans. Networking 5 (1997) 1220-1225

15. Pas, E.I., Principio, S.L.: Braess's paradox: Some new insights. Transpn. Res. B 31 (1997) 265-276

16. Bean, N.G., Kelly, F.P., Taylor, P.G.: Braess' paradox in a loss network. Journal of Applied Probability 34 (1997) 155-159

17. Korilis, Y.A., Lazar, A.A., Orda, A.: Architecting noncooperative networks. IEEE J. Selected Areas in Communications 13 (1995) 1241-1251

18. Korilis, Y.A., Lazar, A.A., Orda, A.: Avoiding the Braess paradox in noncooperative networks. J. Appl. Prob. 36 (1999) 211-222

19. Kameda, H., Altman, E., Kozawa, T., Hosokawa, Y.: Braess-like paradoxes in distributed computer systems. IEEE Trans. Automatic Control 45 (2000) $1687-$ 1691 
20. Kameda, H.: How harmful the paradox can be in the Braess/Cohen-Kelly-Jeffries networks. In: Proc. IEEE INFOCOM 2002, New York (2002)

21. Roughgarden, T.: Designing networks for selfish users is hard. In: Proceedings of the 42nd Annual IEEE Symposium on Foundation of Computer Science. (2001) 472-481 (to appear in a special issue of Journal of Computer and System Sciences).

22. Kameda, H., Pourtallier, O.: Paradoxes in distributed decisions on optimal load balancing for networks of homogeneous computers. J. ACM 49 (2002) 407-433

23. Hsiao, M.T.T., Lazar, A.A.: Optimal decentralized flow control of Markovian queueing networks with multiple controllers. Performance Evaluation 13 (1991) 181-204

24. Parekh, A.K., Gallager, R.G.: A generalized processor sharing approach to flow control in integrated services networks: The single-node case. IEEE/ACM Trans. Networking 1 (1993) 91-10

25. Chakravorti, B.: Optimal flow control of an $\mathrm{M} / \mathrm{M} / 1$ queue with a balanced budget. IEEE Trans. Automatic Control 39 (1994) 1918-1924

26. Korilis, Y.A., Lazar, A.A.: On the existence of equilibria in noncooperative optimal flow control. J. ACM 42 (1995) 584-613

27. Altman, E., Başar, T.: Multiuser rate-based flow control. IEEE Trans. Communications 46 (1998) 940-949

28. Ching, W.K.: A note on the convergence of asynchronous greedy algorithm with relaxation in a multiclass queueing environment. IEEE Communications Letters 3 (1999) 34-36

29. Famolari, D., Mandayam, N.B., Goodman, D., Shah, V.: A new framework for power control in wireless data networks: Games, utility and pricing. In Ganesh, Pahlavan, Zvonar, eds.: Wireless Multimedia Network Technologies. Kluwer Academic Publishers (1999) 289-310

30. Saraydar, C.U., Mandayam, N.B., Goodman, D.J.: Pricing and power control in a multicell wireless data network. IEEE JSAC 19 (2001) 1883-1892

31. Saraydar, C.U., Mandayam, N.B., Goodman, D.J.: Efficient power control via pricing in wireless data networks. IEEE Trans. Communications 50 (2002) 291303

32. Ji, H., Huang, C.Y.: Non-cooperative uplink power control in cellular radio systems. Wireless Networks 4 (1998) 233-240

33. Sung, C.W., Wong, W.S.: A noncooperative power control game for multirate CDMA data networks. IEEE Trans. Wireless Communications 2 (2003) 186-194

34. Kameda, H.: Bounds on benefits and harms of adding connections to noncooperative networks. Technical Report ISE-TR-04-195, Institute of Information Sciences and Electronics, University of Tsukuba (2004)

35. Tantawi, A.N., Towsley, D.: Optimal static load balancing in distributed computer systems. J. ACM 32 (1985) 445-465

36. Kim, C., Kameda, H.: An algorithm for optimal static load balancing in distributed computer systems. IEEE Trans. Comput. 41 (1990) 381-384

37. Kameda, H., Li, J., Kim, C., Zhang, Y.: Optimal Load Balancing in Distributed Computer Systems. Springer (1997)

38. Zhang, Y., Kameda, H., Shimizu, K.: Parametric analysis of optimal load balancing in distributed computer systems. Journal of Information Processing (Info. Proc. Soc. of Japan) 14 (1992) 433-441 